\title{
A Section of Medical Sociology da American Sociological Association completa cinquenta anos e o Journal of Health and Social Behavior comemora
}

\section{The American Sociological Association's Medical Sociology Section turns fifty and the Journal of Health and Social Behavior celebrates}

\author{
Everardo Duarte Nunes \\ Professor colaborador voluntário do Departamento de Medicina Preventiva \\ e Social da Faculdade de Ciências Médicas/Universidade Estadual de Campinas \\ evernunes@uol.com.br
}

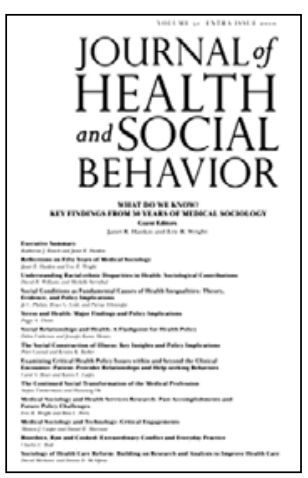

Journal of Health and Social Behavior, Washington, v.51, Suppl. nov. 2010. 159p.
F $m$ 2010, a Section of Medical Sociology da American Sociological EAssociation (ASA) completou cinquenta anos, e o Journal of Health and Social Behavior, que se chamava originalmente Journal of Health and Human Behavior, publicou um número especial para comemorar a data. Nele, revisa muitos aspectos da própria trajetória da sociologia médica. ${ }^{1}$ Como escrevem os editores, são cinquenta anos de dramáticas mudanças nos padrões e no sistema de saúde, sendo o momento para avaliar as contribuições da sociologia para nosso entendimento da doença, prevenção e tratamento. Assim, organizaram, com a colaboração de notáveis cientistas, 11 áreas da sociologia médica a fim de descrever essas contribuições: 1) disparidades étnico-raciais no cuidado à saúde; 2) causas fundamentais das desigualdades em saúde; 3) estresse e saúde; 4) relações sociais e saúde; 5) a construção social da enfermidade; 6) relações

paciente/provedor e comportamentos de busca de ajuda; 7) a transformação social da profissão médica; 8) pesquisas em serviços de saúde; 9) tecnologia; 10) bioética; 11) reforma da saúde. Em todos os artigos, a tônica foi descrever como a sociologia médica criou e testou teorias, mostrando que algumas permaneceram, outras se transformaram e poucas foram rejeitadas.

$\mathrm{O}$ artigo sobre as relações étnico-raciais no cuidado à saúde foi escrito por David R. Williams e Michele Sternthal, da Harvard University, e aborda o tema analisando o entendimento biológico de raça, a ênfase do contexto e da estrutura social como determinantes das diferenças raciais na doença, as múltiplas formas como o racismo afeta a saúde, o papel da história migratória e a posição social na saúde. Os autores recuperam o clássico de William Du Bois, de 1899, The Philadelphia Negro, e assinalam que houve muito pouca atenção dos principais periódicos de sociologia nos EUA sobre a questão da saúde da população negra durante o século XX e que muitas das questões contemporâneas estão presentes nesse trabalho pioneiro. Du Bois apontava que as causas das diferenças raciais eram multifatoriais e primeiramente sociais. Para os autores, o tema se presta claramente ao debate e à ação na arena política, 
mas há necessidade de que os policy makers identifiquem "as barreiras reais e percebidas para implementar iniciativas societárias integrais" (p.S24), a fim de eliminar as diferenças raciais em saúde.

Ainda na temática das desigualdades em saúde, Joe Phelan, Bruce Link e Parisa Tehranifar, da Columbia University, trabalham a noção desenvolvida por Link e Phelan, em 1995, de 'causas fundamentais', que associa status socioeconômico (SSE) e mortalidade, baseada no conceito de 'causas básicas' de Lieberson, de 1985. O modelo comporta quatro componentes: evidência de que os SSE influenciam os resultados de múltiplas doenças; de que os SSE estão relacionados a múltiplos fatores de risco de doenças e morte; de que a utilização de recursos (renda, conhecimento, prestígio, benefícios sociais) desempenha um papel crucial na associação entre SSE e saúde/mortalidade; evidência de que a associação é reproduzida durante o tempo pela substituição de mecanismos intervenientes. Concluem que a associação persiste "apesar do desaparecimento dos fatores de risco e doenças que pareciam explicar a associação" (p.S38), o que sugere a importância do conceito para os estudos da desigualdade em saúde e mortalidade.

Outro tema nesse número é tratado por Peggy Thoits, da Indiana University, e versa sobre as descobertas da relação estresse e saúde e suas implicações para a política de saúde. A autora abre o artigo com o clássico de Selye, de 1956, The Stress of Life, cujos achados com animais em laboratórios sobre reações e estressores seriam confirmados em seres humanos. Destaca o trabalho de Thomas e Rahe, de 1967, quando criaram a Social Readjustment Rating Scale para medir estressores que eram de natureza social, e revisa os desdobramentos que tiveram os estudos sobre 'os eventos da vida' ou 'eventos estressantes'. Dentre os inúmeros pontos levantados, destaca que os sociólogos têm demonstrado o peso das diferenças de raça, gênero, etnia, estado civil, status socioeconômico no desencadeamento do estresse. Ressalta, assim, o papel da estratificação social e das políticas de saúde para apoio e intervenção individual e de programas que visem a intervenções em aspectos estruturais.

As relações (laços) sociais e saúde foram analisadas por Debra Umberson e Jennifer Karas Montez, da University of Texas, que abordam o tema afirmando, inicialmente, que tanto em qualidade como em quantidade elas afetam a saúde mental, o comportamento na saúde, a saúde física e os riscos de mortalidade. Apontam que "Enquanto os relacionamentos são a fonte central de apoio emocional, relacionamentos sociais podem ser extremamente estressantes" (p.S57). Citam, como exemplo, o casamento, que pode ser tanto fonte de apoio como de estresse para os indivíduos. Para as autoras, as relações sociais podem influenciar a saúde por meio de manifestações comportamentais, psicossociais e fisiológicas e não podem ser ignoradas pelas políticas de saúde.

Peter Conrad, da Brandeis University, e Kristin Barker, da Oregon State University, apresentam uma revisão do que consideram a principal perspectiva em sociologia médica - a construção social da doença (illness). Revisam a trajetória histórica do construcionismo, a partir dos anos 1960 e 1970, com Becker e Goffman, passando por Freidson e Foucault. Desenvolvem as ideias tratando dos significados culturais da doença e a experiência da doença como socialmente construída, e assumem que "Por revelar as contingências sociais da doença nos níveis individual, institucional e societário, a abordagem do construcionismo social fornece um valioso instrumento para as formulações das políticas [em saúde]" (p.S76). 
Em importante nota, situam algumas críticas à abordagem, mas esclarecem que, do ponto de vista do construcionismo, deve-se buscar, em primeiro lugar, definir tanto a disease como a illness, e em ambos os casos importa mais a "viabilidade da 'ideia' de uma ou outra, do que a sua validade, per se" (grifos do original) (p.S77).

Examinar as mudanças ocorridas nos últimos cinquenta anos na dinâmica dos 'encontros clínicos', em especial do relacionamento médico/paciente, e acesso ao cuidado à saúde é a proposta de Carol Boyer, da Rutgers, The State University of New Jersey, e Karen Lutfey, dos New England Research Institutes. Embora citem os clássicos sobre o assunto, Renée Fox, Parsons, Reeder e outros, abordam as transformações ocorridas nos papéis dos pacientes, quando se tornam mais ativos e diversificados, pelo fato de basearem o comportamento em riscos, e de as relações paciente/provedor serem de caráter multifacetado, menos paternalistas e mais centradas nos resultados. Para os estudiosos, o cuidado primário é "um setor-chave para as reformas do cuidado à saúde frente a um futuro incerto" (p.S87), mas que tem suas vulnerabilidades, em especial manifestadas pela autonomia dos clínicos. Apontam dois recentes modelos patient-centered medical homes e accountable care organizations para expandir acesso e diminuir custos. Lembram, ainda, que em 2010 foi assinado o Patient Protection and Affordable Act, visando ao cuidado integral.

Sem dúvida, o estudo da profissão médica constitui uma pièce de résistance da sociologia médica e aqui é analisada por Stefan Timmermans e Hyeyoung Oh, da University of California. Além de uma revisão dos principais pontos sobre a profissão nas últimas décadas (comercialização do cuidado médico, controle do próprio trabalho, dominância profissional, desprofissionalização-proletarização-corporatização), mostram que mesmo "as fortes pressões não anunciam o fim da medicina organizada e profissional" (p.S97). São abordados três pontos que desafiam o núcleo do profissionalismo médico: a) as relações consumismo/ interesse profissional/confiança dos pacientes nos provedores de cuidados; b) a medicina baseada em evidências questionando se os profissionais aplicam o conhecimento apropriado; c) o crescimento da indústria farmacêutica e a independência dos profissionais. No artigo, destacam a importância dos sociólogos em documentar a resistência e a aceitação dos médicos às mudanças no sistema de saúde.

Eric Wright, da Indiana University, e Brea Perry, da University of Kentucky, desenvolvem três achados da sociologia dos serviços de saúde: a distribuição desigual dos serviços nos EUA, a reprodução dessas desigualdades pelas organizações de saúde e a configuração da qualidade, efetividade e resultados dos serviços dada pela estrutura das organizações. Destacam que a "fragmentação e falta de coordenação sugerem a necessidade de administração mais centralizada, algo que o mercado de cuidado à saúde não tem sido capaz de alcançar por si mesmo" (p.S115). Concluem que "Quando tomada como um todo, a pesquisa sociológica sobre serviços de saúde destaca a necessidade de um papel mais forte do governo em coordenar e administrar o sistema de saúde dos Estados Unidos" (p.S115).

Tema sobremodo atual - a teconologia em medicina - recebeu atenção de Monica Casper, da Arizona State University, e Daniel Morrison, da Vanderbilt University. Recordam os autores que, no final dos anos 1950, quando foi criada a Medical Sociology Section, da ASA, eram evidentes os avanços tecnológicos em medicina (primeiro marcapasso, produção em massa de antibióticos, primeiro transplante de rim e aperfeiçoamento da hemodiálise 
etc.), ao lado do crescimento dos gastos com saúde (4,5\% do PIB) e do aumento do preço das internações hospitalares. Cinquenta anos depois: aplicação de 16\% do PIB para a saúde; 47 milhões de norte-americanos fora dos planos de seguro; expansão das tecnologias genéticas; nanotecnologia; difusão das pesquisas sobre o genoma etc. Todo esse panorama, sinteticamente denominado biomedicalização, provoca os autores em três pontos: como as tecnologias remodelaram as práticas, reconfigurando o corpo humano e as concepções sobre ele, e seu papel na emergência de novos movimentos sociais na saúde, e apontam as transformações nas teorias que embasaram o conhecimento das tecnologias. Dentre as sugestões de estudos, salientam as relações que o tema mantém com a economia da saúde e das políticas públicas.

Dentro do amplo espectro das temáticas e suas transformações ao longo dos cinquenta anos de sua existência, o Journal of Health and Social Behavior aborda a bioética e seus conflitos na prática clínica e na política de saúde. Trata-se do artigo de Charles Bosk, da University of Pennsylvania, que toma como ponto de partida da análise dois níveis: a linguagem usada nas questões éticas e como se criam as rotinas das organizações e seus procedimentos. Destaco do artigo três pontos referentes às contribuições da sociologia para a bioética: atenção às práticas de pesquisa, pois "sociólogos médicos têm demonstrado como são fracas as habilidades dos pacientes para exercer a autonomia" (p.S142); demonstração de como os complexos contextos influenciam a aplicação dos princípios bioéticos; questionamento da aplicação dos princípios éticos (autonomia, justiça, beneficência, não maleficência) que são "tão bem adaptados aos problemas individuais" (p.S143) podem ser estendidos à população.

$\mathrm{O}$ artigo que encerra esse número do Journal of Health and Social Behavior apresenta um trabalho com o sugestivo nome de "Sociology of health care reform: building on research and analysis to improve health care" (Sociologia da reforma dos cuidados de saúde: a pesquisa e a análise na construção do cuidado à saúde), de autoria de David Mechanic, da Rutgers, The State University of New Jersey, e Donna McAlpine, da University of Minnesota. Ao considerar que o sistema de saúde norte-americano é amplo, caro e complexo, dizem que a sociologia médica tem desempenhado papel significativo na compreensão dos diversos aspectos econômicos, profissionais e ideológicos que influenciam a organização, a provisão e as reformas desse sistema. Destacam não somente os estudos sobre microprocessos (relações internas), mas os que se voltam para as políticas de saúde. Centralizam a apresentação em cinco temas que tratam de usos e custos na provisão de cuidados, modalidades de reembolso, características culturais das organizações de saúde, retórica e real tomada de decisões sobre o paciente, compartilhamento de informações e a veracidade das mesmas. Acentuam que as reformas se baseiam em confiança e cooperação entre as organizações e as associações profissionais.

Procurou-se nesta resenha destacar pontos considerados fundamentais de textos que apresentam revisões calcadas em extensa, mas seleta, bibliografia. Os autores não apenas retomam os textos, mas analisam, sistematizam e sugerem extensões dos temas a muitos problemas atuais e futuros no campo da saúde. Ao contextualizar os seus estudos, colocam em evidência as transformações ocorridas no quadro epidemiológico e os problemas não resolvidos pelo sistema de saúde norte-americano durante meio século (por exemplo, milhões de pessoas sem seguro-saúde, altos custos do sistema etc.), convocando os cientistas sociais para o aprofundamento das análises necessárias no sistema e nas relações internas a ele. 
Com fator de impacto de 2,722; 11 a entre 131 publicações classificadas em Public, Environmental \& Occupational Health, pela agência Thomson Reuters, o Journal of Health and Social Behavior contribui com importantes abordagens aos problemas coletivos de saúde da sociedade americana, no momento em que ela clama por reformas nesse setor.

\section{NOTA}

${ }^{1}$ A história da Seção de Sociologia Médica e do Journal é relatada por Bloom (2002). O Conselho de Sociologia da ASA aprovou a criação da seção em setembro de 1959, e, no mês seguinte, August Hollingshead foi eleito seu primeiro presidente. O Journal of Healh and Human Behavior foi criado em 1960, como publicação independente, sendo considerado "o primeiro periódico nos tempos modernos dedicado inteiramente à sociologia médica" (Bloom, 2002, p.224). O nome do periódico foi mudado para Journal of Health and Social Behavior e, em março de 1966, começou a ser publicado pela ASA. Seu primeiro editor, escolhido pela ASA, foi Eliot Freidson.

\section{REFERÊNCIA}

BLOOM, Samuel W.

The world as scalpel: a history of medical sociology. New York: Oxford University Press.

2002. 